\title{
Analytical Transient Response and Propagation Delay Model for Nanoscale CMOS Inverters
}

\author{
Y. Wang, and M. Zwolinski \\ School of Electronics and Computer Science, University of Southampton, \\ Southampton, SO17 1BJ, UK, \\ E-mail:yw2@ecs.soton.ac.uk, mz@ecs.soton.ac.uk
}

\begin{abstract}
This paper presents a new analytical propagation delay model for a nanoscale CMOS inverter. By using a nonsaturation current model, the analytical input-output transfer responses and propagation delay model are derived. The model is used for calculating inverter delays with different input transition times, load capacitances and supply voltages. Delays predicted by proposed model are in good agreement with that of transistor level simulation results from SPICE, and errors are less than $3 \%$.
\end{abstract}

\section{INTRODUCTION}

As one of the most important performance parameters in CMOS digital circuits, the propagation delay is of concern to designers and users. A circuit's speed/frequency and dynamic power dissipation are both affected significantly by propagation delay, and hence timing analysis has been investigated for several decades [1-7]. With the increasing complexity of modern very large scale integration (VLSI) systems, transistor level simulation consumes much more computation time because of the nonlinear transfer characteristics of CMOS gates [8-10]. Therefore, an analytical delay model that does not need numerical iterations is needed to extract delay efficiently, and much work has been published on the topic $[3,6-$ 19].

For extracting the propagation delay, development of a delay model for a CMOS inverter is considered as the first step [14], and a number of inverter delay models have been developed [6-15]. The first inverter delay expression was introduced by Burns [1]. Early models were based on Shockley's square law MOSFET model which does not include the carrier velocity saturation effect $[1,2]$. As the drain current $\left(I_{d s}\right)$ deviates significantly from the Shockley model in the submicron region, Sakurai et al. [3] proposed a model using an $\alpha$-power law current model which includes the carrier velocity saturation effect of short channel devices. Several analytical delay expressions based on the $\alpha$-power law model were introduced thereafter [10, 13]. However, in modern small dimension MOSFETs, $\mathrm{I}_{\mathrm{ds}}$ does not show saturation [20-22]. Therefore, $\alpha$-power law based delay models would underestimate inverter propagation delay by using higher $I_{d s}$ (at $V_{d s}=V_{d d}$ ) as the saturation current in the nominal saturation region.

On the other hand, some delay models did not take the current through the loading transistor into account $[3,13]$, including both the overshooting and short-circuit current $[10$, 14, 23]. Moreover, with continuous scaling down of transistor dimensions, the charging/discharging of input-output coupling capacitance $\left(C_{M}\right)$ inevitably affects inverter characteristics and propagation delays, which should be considered in developing delay models [7, 8, 10, 12, 14, 16, 23].

The reported propagation delay expressions for a submicron inverter are complex [8-10, 14], which limits the exten- sive use of them. The objective of this work is to develop a new analytical propagation delay model for a Nanoscale CMOS inverter. A non-saturation $I_{d s}$ model for a MOSFET is proposed, and the effects of $I_{o v}$ and $C_{M}$ are considered. The analytical expression of the output response is derived by solving differential equations in each transition region, giving the propagation delay of a CMOS inverter.

\section{NON-SATURATION DRAIN CURRENT MODEL AND INVERTER TRANSIENT RESPONSE}

\section{A. PROPOSED DRAIN CURRENT MODEL FOR NANO MOSFETS}

The MOSFET output characteristics are simulated in SPICE using the BSIM4 model. The model parameters were extracted from $35 \mathrm{~nm}$ technology bulk devices with an oxide thickness of $0.86 \mathrm{~nm}$. Fig. 1(a) shows the output characteristics of an n-MOSFET with both width and length of $35 \mathrm{~nm}$; $I_{d s}$ predicted by an $\alpha$-power law model is also shown. The non-saturation phenomena and obvious discrepancy between the $\alpha$-power law and simulation are observed.

It is seen that $I_{d s}$ shows piecewise linearity versus $V_{d s}$, the first segment corresponds to the triode region and the second is observed before the substrate current induced body effect takes effect. Linear fitting to the second segment is shown in Fig. 1(a), and linear equations are used in describing the driving current of dynamic inverter behaviour.

During a rising input transient, $V_{d s}\left(=V_{\text {out }}\right)$ of the driving nMOSFETs varies with gate voltage $\left(V_{g s}=V_{i n}\right)$, so $I_{d s}$ lies on different lines in Fig. 1(a). To obtain $I_{d s}$ at any transition point, linear equations for the second region at any $V_{g s}$ should be known, which can be derived by the two-point method. For example, two simulated or measured points are taken to achieve the objective at $V_{g s}=V_{d d}$, one is $I_{d s 0}\left(I_{d s}\right.$ at $\left.V_{g s}=V_{d s}=V_{d d}\right)$, and the other is chosen at the near transition point of $I_{d s}-V_{d s}$ such as $I_{d s 0}^{\prime}\left(I_{d s}\right.$ at $\left.V_{g s}=2 V_{d s}=V_{d d}\right)$. For the case of $V_{g s}$ being less than $V_{d d}$, the two points can be estimated by the $\alpha$-power law as illustrated in Fig. 1(b).
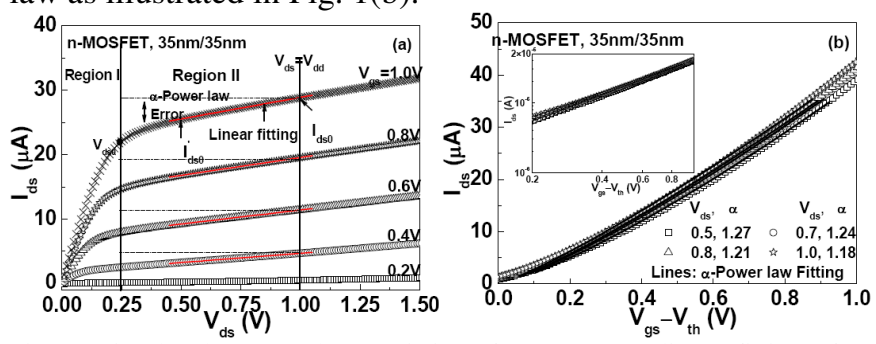

Fig. 1. Simulated output characteristics of n-MOSFET, linear fitting of $I_{d}$ from $V_{d s}=0.5 V_{d d}$ to $V_{d d}$ are shown (a). $\alpha$-power fitting of $I_{d s} \sim\left(V_{g s}-V_{t h}\right)$, inset is $\log -\log$ plot of the same group data, $\alpha \sim 1.2$ for Nano MOSFETs (b). 
It is found that $I_{d s}$ is proportional to $\left(V_{g s}-V_{t h}\right)^{\alpha}$ from Fig. 1(b), where $\alpha$ is about 1.2 (which is reasonable for submicron devices [3]). Therefore, $I_{d s}$ in region II of Fig. 1(a) can be expressed as follows:

$I_{d s}=I_{d s 0}\left(\frac{V_{g s}-V_{t h}}{V_{d d}-V_{t h}}\right)^{\alpha}+2\left(I_{d s 0}-I_{d s 0}\right)\left(\frac{V_{g s}-V_{t h}}{V_{d d}-V_{t h}}\right)^{\alpha} \frac{V_{d s}-V_{d d}}{V_{d d}}$

$\mathrm{V}_{\text {th }}$ is the threshold voltage, $\alpha$ is the fitting parameter reflecting velocity saturation effects. Eq. (1) will be used in deriving the inverter analytical propagation delay model.

\section{B. CMOS INVERTER SWITCHING CHARACTERISTICS ANALYSIS}

The schematic of a CMOS inverter is shown in Fig. 2(a). The ratio of the channel widths $(W)$ between p-MOSFET and n-MOSFET is set at 1.74. $C_{L}$ includes output capacitance and load capacitance, $C_{M}$ is input-to-output coupling capacitance which is given as follows:

$$
C_{M}=C_{o x}\left(\frac{W_{p e f f} L_{p e f f}}{2}+L_{D p} W_{p e f f}+L_{D n} W_{n e f f}\right)
$$

where $W_{\text {peff }}$ and $W_{\text {neff }}$ are the effective channel widths of the pMOSFET and n-MOSFET, respectively. $L_{\text {peff }}$ is the effective channel length of the p-MOSFET; $L_{D p}$ and $L_{D n}$ are the gatedrain under-diffusion of p-MOSFET and n-MOSFET.

The dynamic behaviour of the inverter can be derived from Kirchhoff's current law at the output node:

$$
C_{L} \frac{d V_{\text {out }}}{d t}=C_{M} \frac{d\left(V_{\text {in }}-V_{\text {out }}\right)}{d t}+I_{p}-I_{n}
$$

where $V_{\text {out }}$ and $V_{\text {in }}$ are the output and input voltages, $I_{p}$ and $I_{n}$ are the $I_{d s}$ values of the p-MOSFET and n-MOSFET, respectively. In this work, the differential equation is solved for a rising input where the n-MOSFET is the driving transistor and the p-MOSFET is the loading transistor. The rising input signal is expressed as:

$V_{\text {in }}=0$ at $t \leq 0, V_{i n}=V_{d d} \times t / t r$ for $0 \leq t \leq t r$ and $V_{i n}=V_{d d}$ at $t \geq t r$,

where $t r$ is the riseime. Therefore, the propagation delay, tpHL (high to low) which is the time interval from $V_{\text {in }}=50 \% V_{d d}$ to $V_{\text {out }}=50 \% V_{d d}$ will be derived.
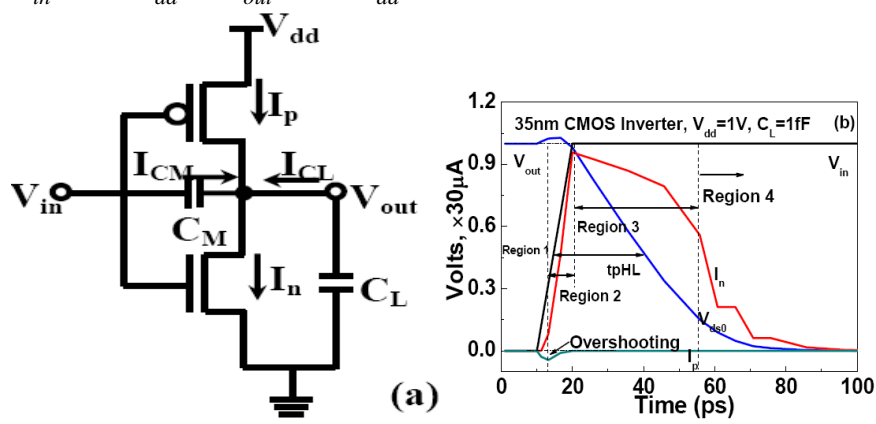

Fig. 2. (a) A CMOS inverter discharging model, (b) input-output voltage and current evolution for a linear rising input signal, inverter operation regions and $t p H L$ definition are shown.

Fig. 2(b) shows both the input-output relation and $I_{p}$ and $I_{n}$ for a rising input case. $I_{p}$ is negative at the beginning of the transition because of overshooting $[10,14]$. The overshooting time $\left(t_{o v}\right)$ cannot be ignored in nanoscale digital circuits [23]. $I_{p}$ becomes positive after n-MOSFET turns on, which called the short-circuit current but it is ignored for fast input ramps.

For a fast rising input ramp, the trajectory of $V_{\text {out }}$ is shown by arrowed lines in Fig. 3. The driving current $\left(I_{n}\right)$ is estimated as follows:

Region $1, I_{n} \approx I_{\text {subn }}, \quad 0 \leq t \leq V_{t h n} \times t r / V_{d d}, \quad 0 \leq V_{i n} \leq V_{t h n}$, cut-off and subthreshold region.
Region 2, $I_{n}=I_{d s 0}\left(\frac{V_{g s}-V_{t h}}{V_{d d}-V_{t h}}\right)^{\alpha}, V_{t h n} \times t r / V_{d d} \leq t \leq t r, V_{t h n} \leq V_{i n} \leq V_{d d}$, Region II in Fig. 1(a) at $V_{d s}=V_{d d}$.

Region 3, $I_{n}=I_{d s 0}+2\left(I_{d s 0}-I_{d s 0}^{\prime}\right) \frac{V_{d s}-V_{d d}}{V_{d d}} t \geq t r$, Region II in Fig. 1(a) at $V_{g s}=V_{d d}$ and $V_{d s} \geq V_{d s 0}$.

Region $4, I_{n}=\beta\left(V_{d d}-V_{t h}-\frac{1}{2} V_{d s}\right) V_{d s}, t \geq t r$, Region I in Fig. 1(a) at $V_{g s}=V_{d d}$ and $V_{d s} \leq V_{d s 0}$.

where $I_{s u b n}$ is the subthreshold current, $V_{g s n}=V_{i n}, V_{d s n}=V_{\text {out }}$, $\beta=W_{n} \mu_{e} C_{o x n} / L_{n}$ and $\mu_{e}$ is the electron mobility.
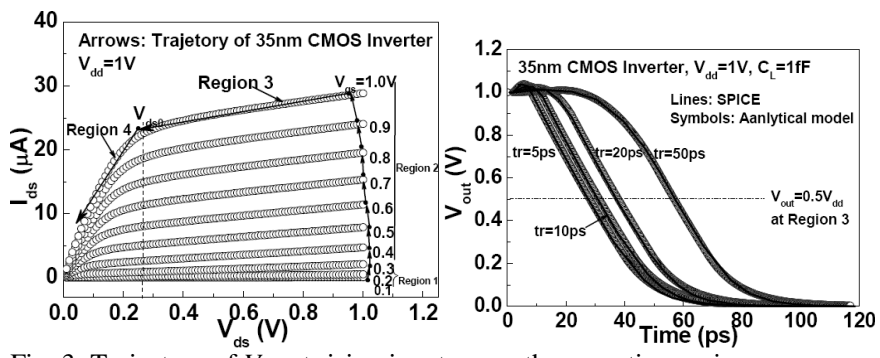

Fig. 3. Trajectory of $V_{\text {out }}$ at rising input ramp, the operation regions are corresponding to that of Fig. 2(b)'s.

Fig. 4. Comparison of $V_{\text {out }}$ between predictions of the model and transistor level simulations of SPICE, with input rising time increases from $5 \mathrm{ps}$ to $50 \mathrm{ps}$.

\section{ANALYTICAL INVERTER TRANSIENT RE- SPONSE ANALYSIS AND PROPAGATION DELAY}

\section{A. ANALYTICAL SOLUTIONS OF INVERTER TRAN- SIENT RESPONSE}

By connecting the switching behavioural of CMOS inverter in Fig. 2(b) and the suggested driving current model, corresponding forms of Eq. (3) can be rewritten and solved as follows.

Region 1: $0 \leq \leq \leq V_{t h n} \times t r / V_{d d}, 0 \leq V_{i n} \leq V_{t h n}$

The n-MOSFET operates in the subthreshold region and the p-MOSFET in the linear region with $V_{d s p}>0$ because of effects of the gate-to-drain coupling capacitance. Since expressions of the overshooting and subthreshold current are complex, one cannot solve analytically the differential equation for this region. So the average value of $I_{s u b n}\left(\right.$ at $\left.V_{i n}=V_{t h n} / 2\right)$ is used to substitute for $I_{n}$ and $I_{p}$. Therefore, Eq. (3) is:

$C \frac{d V_{\text {out }}}{d t}=C_{M} \frac{d V_{\text {in }}}{d t}-2 I_{\text {subn }}=C_{M} \frac{V_{d d}}{t r}-2 I_{\text {subn }}$

where $C=C_{L}+C_{M}$, considering the boundary condition of $V_{\text {out }}=V_{d d}$ at $t=0$,

$V_{\text {out } 1}=\left(\frac{C_{M} V_{d d}}{C t r}-\frac{2 I_{\text {subn }}}{C}\right) t+V_{d d}$

\section{Region 2, $V_{t h n} \times t r / V_{d d} \leq \leq t r, V_{t h n} \leq V_{i n} \leq V_{d d}$}

The n-MOSFET switches on and $V_{d s n}\left(V_{\text {out }}\right)$ stays at almost $V_{d d}$ for fast input ramps cases, $I_{n}$ is calculated by $\alpha$-power law. p-MOSFET current equals approximately to zero because $\mathrm{V}_{\mathrm{dsp}}$ is almost zero. The differential equation is:

$C \frac{d V_{\text {out }}}{d t}=C_{M} \frac{V_{d d}}{t r}-I_{d s 0}\left(\frac{V_{i n}-V_{t h n}}{V_{d d}-V_{t h n}}\right)^{\alpha}$

The solution is obtained by using the boundary condition of $V_{\text {out } 2}\left(t=V_{\text {thn }} \times t r / V_{d d}\right)=V_{\text {out } 1}\left(t=V_{\text {thn }} \times t r / V_{d d}\right)$ :

$V_{\text {out } 2}=\frac{\left(t / t r-V_{t h n}\right)^{\alpha+1}}{\left(V_{d d}-V_{t h n}\right)^{\alpha}}\left(\frac{t r}{C(\alpha+1)}\right)+\frac{C_{M} V_{d d} t}{C t r}+V_{d d}-\frac{2 V_{t h n} I_{\text {subn }}}{C} t r$ 
Region 3, $t \geq r, V_{i n}=V_{d d}$ and $V_{o u t} \geq V_{d s 0}$.

The n-MOSFET operates in linear region II, $V_{g s n}=V_{i n}=V_{d d}$. p-MOSFET turns off. The dynamic behaviour is described by:

$$
C \frac{d V_{\text {out }}}{d t}=-I_{d s 0}-2\left(I_{d s 0}-I_{d s 0}^{\prime}\right) \frac{V_{o u t}-V_{d d}}{V_{d d}}
$$

The above first order differential equation can be solved analytically with $V_{\text {out } 3}=V_{\text {out } 2}$ at $t=t r$.

$V_{\text {out } 3}=A_{1}+\left(A_{2}-A_{3}-A_{1}\right) e^{A_{4}(t-t r)}$

where $A_{1}=\frac{2 I_{d s 0}^{\prime}-I_{d s 0}}{2\left(I_{d s 0}^{\prime}-I_{d s 0}\right)} V_{d d}, A_{2}=\left(1+\frac{C_{M}}{C}\right) V_{d d}-\frac{2 I_{s u b n} V_{t h n}}{C V_{d d}} t r$,

$$
A_{3}=\frac{I_{d s 0}\left(V_{d d}-V_{t h n}\right)}{C V_{d d}(\alpha+1)} t r, A_{4}=\frac{2\left(I_{d s 0}^{\prime}-I_{d s 0}\right)}{C V_{d d}}
$$

Region 4, $t \geq r, V_{\text {in }}=V_{d d}$ and $V_{\text {out }} \leq V_{d s o}$.

The n-MOSFET operates in the linear region I of Fig. 1(a), $V_{g s n}=V_{i n}=V_{d d}$ and p-MOSFET is off.

$$
C \frac{d V_{o u t}}{d t}=-\beta\left(V_{d d}-V_{t h}-\frac{1}{2} V_{d s}\right) V_{d s}
$$

The equation can be simplified by neglecting the second order term. By continuity conditions,

$V_{\text {out } 4}=V_{d s 0} \exp \left(-B t_{0}\right) \exp (B t)$

$B=\beta\left(V_{t h}-V_{d d}\right) / C, t_{0}$ is calculated from Eq. (9) at $V_{\text {out } 3}=\mathrm{V}_{\mathrm{ds} 0}$.

\section{B. INVERTER PROPAGATION DELAY EVALUATION}

The high-to-low propagation delay $(t p H L)$ is calculated by: $t p H L=t_{\mathrm{v}_{\text {out }}=50 \% \mathrm{v}_{\mathrm{dd}}}-0.5 \mathrm{tr}$

The $50 \% V_{d d}$ level of $V_{\text {out }}$ always occurs in region 3 for fast input ramp cases, and $t p H L$ is derived from Eq. (9),

$$
t p H L=\frac{1}{A_{4}} \ln \left(\frac{0.5 V_{d d}-A_{1}}{A_{2}-A_{3}-A_{1}}\right)+\frac{1}{2} t r
$$

For very slow input transitions, $V_{\text {out }}=0.5 V_{d d}$ would occur in region 2, tpHL can be obtained from Eq. (7). The parameters in the $t p H L$ formula can be obtained from either simulation or measurement. Both the overshoot current of the p-MOSFET and subthreshold current of the n-MOSFET are taken into account. The non-saturating driving current is considered for nano MOSFETs, which reduces the delay errors compared with models using a saturated driving current $[3,10,13]$.

\section{RESULTS AND DISCUSSIONS}

In this section, $V_{\text {out }}$ and $t p H L$ calculated by the proposed model are compared with those obtained from SPICE. The physical and electrical parameters of both transistors are listed in table I.

Table. I. MOSFET PHYSICAL AND ELECTRICAL PARAMETERS USED IN CALCULATIONS OF PROPAGATION DELAY

\begin{tabular}{|c|c|c|c|c|}
\hline Device & $\mathrm{L}(\mathrm{nm})$ & $\mathrm{W}(\mathrm{nm})$ & $\begin{array}{c}\mathrm{I}_{\mathrm{ds} 0} \\
\left(\mathrm{~V}_{\mathrm{gs}}=\mathrm{V}_{\mathrm{ds}}=1\right)\end{array}$ & $\begin{array}{c}\mathrm{I}_{\mathrm{ds} 0}^{\prime} \\
\left(\mathrm{V}_{\mathrm{gs}}=2 \mathrm{~V}_{\mathrm{ds}}=1\right)\end{array}$ \\
\hline $\mathbf{n}-\mathrm{MOS}$ & $\mathbf{3 5}$ & $\mathbf{3 5}$ & $\mathbf{2 8 . 8 4} \boldsymbol{A}$ & $\mathbf{2 5 . 4 6 \mu A}$ \\
\hline P-MOS & 35 & 61 & $28.84 \mu \mathrm{A}$ & $25.46 \mu \mathrm{A}$ \\
\hline $\mathrm{I}_{\text {sub(ave) }}$ & $\mathrm{V}_{\text {th }}(\mathrm{V})$ & $\alpha$ & $\mathrm{C}_{\mathrm{ox}}(\mathrm{F})$ & $\mathrm{C}_{\mathrm{gdo}}(\mathrm{F})$ \\
\hline $\mathbf{0 . 5 4} \mu \mathrm{A}$ & $\mathbf{0 . 2 6 1 4 8}$ & $\mathbf{1 . 2}$ & $\mathbf{0 . 0 4 9 2 f}$ & $\mathbf{0 . 0 2 1 6 f}$ \\
\hline $0.54 \mu \mathrm{A}$ & -0.26148 & 1.2 & $0.0857 \mathrm{f}$ & $0.0376 \mathrm{f}$ \\
\hline
\end{tabular}

Fig. 4 is a comparison of $V_{\text {out }}$ from calculation and simulation. $V_{d d}$ and $C_{L}$ are $1 \mathrm{~V}$ and $1 \mathrm{fF}$, respectively, with input rising time varying from $5 \mathrm{ps}$ to $50 \mathrm{ps}$. It is shown that the proposed model results are very close to that produced by SPICE, which means the approximation of the currents and the derivation is good.
Fig. 5 shows $t p H L$ at different $t r(\leq 50 \mathrm{ps})$ with $V_{d d}=1 \mathrm{~V}$ and $C_{L}=1 \mathrm{fF}$. The input transition time has little effect on the currents $\left(I_{o v}, I_{s u b n}\right.$ and $I_{d s n}$ at $\left.V_{g s n}=V_{d d}\right)$, and $t p H L$ is calculated by using the parameters in Table I. Simulated $t p H L$ is plotted for comparison; it is observed that the two set of results are close to each other with an average error of about $1.43 \%$. The calculated $t p H L$ from the analytical model of Ref. [3] is also given, from which significant deviations from the simulated results are observed. Inaccuracies of reported models may result from ignoring $I_{o v}, I_{s u \mathrm{~b}}$ and the coupling capacitance, as well as from using a constant driving current.

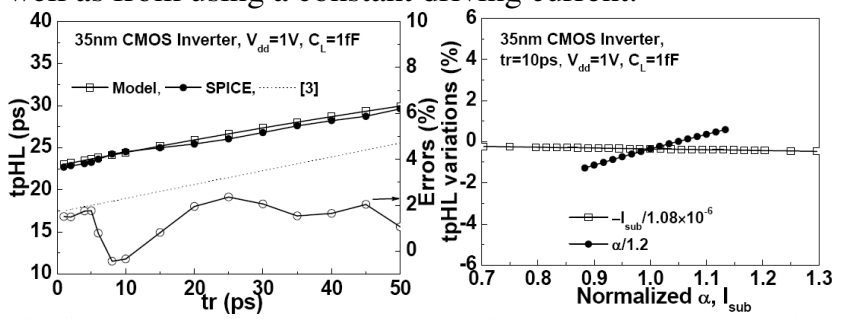

Fig. 5. $t p H L$ calculated by proposed analytical model are compared with that simulated by SPICE with $t r$ varying, the average error is acceptable. $t p H L$ predicted by previous model are also plotted for comparison.

Fig. 6. The calculated $t p H L$ are not affected significantly by variations of delay model parameters of $I_{\text {sub }}$ and $\alpha$.

It is noticed that variations of average $I_{\text {sub(ave) }}$ do not give the variation of $t p H L$ shown in Fig. 6. One of the reasons is the active time of $I_{o v}$ and $I_{s u b}$ is shorter during switching. On the other hand, the range of $\alpha$ in Fig. 1(b) does not result in significant fluctuations of $t p H L$. Therefore, the usability of proposed model is increased.

Fig. 7 shows $t p H L$ as a function of $C_{L}$ at $V_{d d}=1 \mathrm{~V}$ and $t r=10$ ps. $C_{L}$ affect $I_{o v}$ but its effects on the driving current is negligible. The discrepancies between the proposed analytical model and simulation results are negligible, with errors within $3 \%$. Application of this analytical model on different $C_{L}$ is useful for extending its use in more complex digital circuits by the "collapsing" technique, which reduces gates to equivalent inverters [16].

$V_{d d}$ decreases continuously with MOSFET dimension scaling. Lower $V_{d d}$ has the advantages of lowering power dissipation and reducing high electric field effects. However, CMOS intrinsic delay increases rapidly with $V_{d d}$ decreasing if $V_{t h}$ does not reduce in proportion to $V_{d d}$, which is mainly due to the loss of large-signal transcondutance $\left(I_{d s} / V_{d d}\right)$ [24]. The adverse effects are alleviated to some extent by lowering $V_{t h}$. Nevertheless, $V_{t h}$ cannot be always reduced in proportion to $V_{d d}$ in view of subthreshold current (and hence the power) as well as gate oxide electric field. Therefore, delays increase at lower $V_{d d}$.

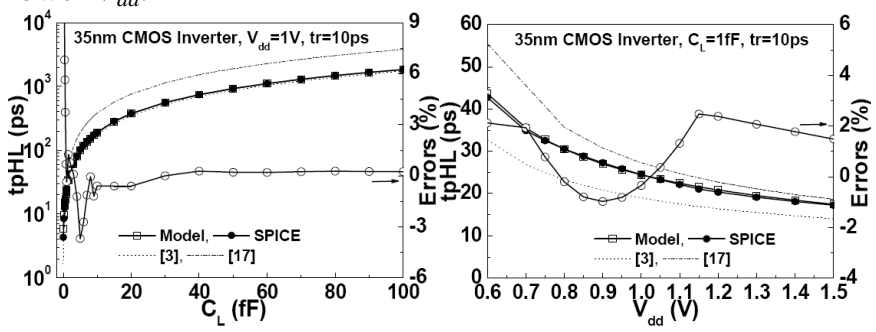

Fig. 7. Comparison of calculated and simulated $t p H L$ at different load capacitance, the average error is about $1.08 \%$. Delays calculated by previous models are also shown.

Fig. 8. The proposed inverter propagation delay model can be used at relative larger range of $V_{d d}$, with errors from SPICE simulation are not more than $2.5 \%$. The predictions of previous analytical models are shown for comparison.

Fig. 8 shows SPICE simulations and model predictions of tpHL versus $V_{d d}$ at $t r=10 \mathrm{ps}$ and $C_{L}=1 \mathrm{fF}$. The errors are 
smaller than $2.5 \%$ when $V_{d d}$ is in the range 0.6 to $1.5 \mathrm{~V}$, which confirms the validity of the proposed analytical model. It should be pointed that $I_{d s 0}$ and $I_{d s 0}^{\prime}$ are different from the values of Table I when $V_{d d}$ is not equal to $1.0 \mathrm{~V}$, and the currents are simulated at $V_{g s}=V_{d s}=V_{d d}$ and $V_{g s}=2 V_{d s}=V_{d d}$, respectively.

tpHL calculated by reported analytical models are compared for different cases in Figs. 5, 7 and 8. As analysed above, $\alpha$-power law based models $[3,10,13]$ use $I_{d s 0}$ as the saturation current, which is larger than the actual driving current and they underestimate $t p H L$. On the contrary, the model of [17] takes the inverter switching trajectory from $V_{d s}=V_{d d}=2 V_{g s}\left(I_{d s}=I_{L}\right)$ to $V_{g s}=2 V_{d s}=V_{d d}\left(I_{d s}=I_{H}\right)$ which is not suitable for a nano inverter. The method of taking half the sum of $I_{H}$ and $I_{L}$ as the effective current in [17] underestimates the driving current and predicts higher delays. To the best of our knowledge, the analytical delay expression presented in this work has the advantages of simple, accurate and operable for Nanoscale CMOS digital circuits compared with the reported models.

\section{CONCLUSIONS}

For nanoscale MOS transistors, the drain current in the saturation region does not show saturation characteristics any more. Therefore, reported propagation delay models, such as the series of $\alpha$-power models, underestimate the delays of nano CMOS inverters. This paper developed an analytical propagation delay model based on a $35 \mathrm{~nm}$ technology CMOS inverter. The effects of non-saturation current, input-to-output coupling capacitance, overshooting current and subthrehsold current are considered in proposed model.

The input waveform slope, load capacitance and supply voltage significantly affect inverter delay, which is predicted by the presented model. The accuracy and validity of the model is verified by comparing with transistor level simulation results of SPICE, with acceptable errors (less than 3\%) at large ranges of input transition times, load capacitances and supply voltages.

We presented accurate and direct analytical expressions of output transient response and propagation delays for a nanoscale CMOS inverter. By simplifying more complex digital gate circuits to an equivalent inverter, this model will be valuable in supplying accurate and fast estimated delays for designers.

\section{ACKNOWLEDGEMENTS}

This work is supported by the Engineering and Physical Sciences Research Council (EPSRC), United Kingdom under grant No. EP/E002064/1. The authors are grateful to the Device Modelling Group, University of Glasgow for the model cards.

\section{REFERENCES}

1. J. R. Burns, "Switching response of complementary-symmetry MOS transistor logic circuit," RCA Rev., vol. 25, pp. 627-661, 1964. 2. N. Hedenstierna, and K. O. Jeppson, "CMOS circuit speed and buffer optimization," IEEE Trans. Computer-Aided Design of integrated circuits and systems, vol. 6, pp. 270-281, 1987.

3. T. Sakurai and A. R. Newton, "Alpha-power law MOSFET model and its applications to CMOS inverter delay and other formulas," IEEE J. Solid-state circuits, vol. 25, pp. 584-594, 1990.

4. L. Z. Zhang, W. Chen, Y. Hu, and C. C. Chen, "Statistical static timing analysis with conditional linear MAX/MIN approximation and extended canonical timing model," IEEE Trans. Computer-
Aided Design of integrated circuits and systems, vol. 25, pp. 11831191, 2006.

5. B. Cline, K. Chopra, D. Blaauw, A. Torres, and S. Sundareswaran, "Transistor-specific delay modelling for SSTA," Design, automation and test in Europe, pp. 592-597, 2008

6. S-H Jung, J-H Baek, and S-Y Kim, "Short circuit power estimation of static CMOS circuits," Proceedings of the conference on Asia south pacific design automation, pp. 545-550, 2001.

7. A. A. Hamoui, and N. C. Rumin, "An analytical model for current, delay, and power analysis of submircon CMOS logic circuits," IEEE Trans. Circuits and systems-II: Analog and digital signal processing, vol. 47, pp. 999-1007, 2000.

8. P. Liu, and Y. J. Lee, "An Accurate Analytical Propagation Delay Model of Nano CMOS Circuits", IEEE International SoC Design Conference (ISOCC), pp. 200-203, 2007.

9. P. Cocchini, G. Piccinini, and M. Zamboni, "A comprehensive submicrometer MOST delay model and its application to CMOS buffers," IEEE J. Solid-state circuits, vol. 32, pp. 1254-1262, 1997.

10. L. Bisdounis, S. Nikolaidis, and O. Koufopavlou, "Analytical transient response and propagation delay evaluation of CMOD inverter for short-channel devices," IEEE J. Solid-state circuits, vol. 33, pp. 302-306, 1998.

11. A. N-Lishi, and N. C. Rumin, "Simultaneous delay and maximum current calculation in CMOS gates," Electronics letters, vol. 28, pp. 682-684, 1992.

12. K. O. Jeppson, "Modeling the influence of transistor gain ratio and the input-to-output coupling capacitance on the CMOS inverter delay," IEEE J. Solid-state circuits, vol. 29, pp. 646-654, 1994.

13. S. Dutta, S. S. M. Shetti, and S. L. Lusky, "A comprehensive delay model for CMOS inverters," IEEE J. Solid-state circuits, vol. 30, pp. 864-871, 1995.

14. J. L. Rossello, and J. Segura, "An analytical charge-based compact delay model for submicrometer CMOS inverters," IEEE Trans. Circuits and systems-I: Regular papers, vol. 51, pp.1301-1311, 2004 15. J. Chang, and L. G. Johnson, "A Novel Delay Model of CMOS VLSI Circuits," IEEE International Midwest Symposium on Circuits and Systems, vol. 2, pp.481-485, 2006.

16. A. N-Lishi, and N. C. Rumin, "Inverter models of CMOS gates for supply current and delay evaluation," IEEE Trans. ComputerAided Design of integrated circuits and systems, vol. 13, pp. 12711279, 1994.

17. M. H. Na, E. J. Nowak, W. Haensch, and J. Cai, "The effective drive current in CMOS inverters," IEEE International Electron devices meeting, pp. 121-124, Dec. 2002.

18. K. Kasamsetty, M. Ketkar, and S. S. Sapatnekar, "A new class of convex functions for delay modelling and its application to the transistor sizing problem," IEEE Trans. Computer-Aided Design of integrated circuits and systems, vol. 19, pp. 779-788, 2000.

19. T-W Chiang, C. Y. R. Chen, and W-Y Chen, "An efficient gate delay model for VLSI design," Proceedings of IEEE International conference on computer design (ICCD), pp. 450-455, 2007.

20. J. B. Roldan, F. Gamiz, J. A. L-Villanueva, P. Cartujo, and J. E. Carceller, "A model for the drain current of deep submicrometer MOSFET's including electron velocity overshoot," IEEE Trans. Electron Device, vol. 45, pp. 2249-2251, 1998.

21. K. Y. Lim, and X. Zhou, "An analytical effective channel-length modulation model for velocity overshoot in submicron MOSFETs based on energy-balance formulation", Microelectronics Reliability, vol. 42, pp. 1857-1864, 2002.

22. A. Benfdila, and F. Balestra, "On the drain current saturation in short channel MOSFETs," Microelectronics Journal, vol. 37, pp. 635-641, 2006.

23. Z. C. Huang, H. Yu, A. Kurokawa, and Y. Inoue, "Modeling the Overshooting Effect for CMOS Inverter in Nanometer Technologies," Asia and South Pacific Design Automation Conference, pp. 565-570, 2007.

24. Y. Taur, and T. H. Ning, Fundamentals of modern VLSI devices, Press syndicate of the university of Cambridge, 1998. 